26. M. Ramkumar and A. N. Akansu, IEEE 2nd Workshop Multimedia Signal Process. Dec. 1998, pp. 267-272.

27. M. Ramkumar and A. N. Akansu, SPIE Multimedia Syst. Appl. 3528, 482-492 (1998).

28. S. D. Servetto, C. I. Podilchuk, and K. Ramachandran, IEEE Int. Conf. Image Process. 1, 445-448 (1998).

29. A. Cohen and A. Lapidoth, Proc. Int. Symp. Inf. Theory, June 2000, p. 48.

30. P. Jessop, Int. Conf. Acoust. Speech Signal Process., 80, $2,077-2,080$.

31. F. Mintzer and G. W. Braudaway, Int. Conf. Acoust. Speech Signal Process. 80, 2,067-2,070.

32. M. Holliman, N. Memon, and M. M. Yeung, SPIE Security and Watermarking of Multimedia Contents, Jan. 1999, pp. 134-146.

33. F. Hartung, J. K. Su, and B. Girod, SPIE Security and Watermarking of Multimedia Contents, 1999, pp. 147-158.

34. J. Dittmann et al., SPIE Security and Watermarking of Multimedia Contents, 1999, pp. 171-182.

35. J. Fridrich and M. Goljan, SPIE Security and Watermarking of Multimedia Contents, 1999, pp. 214-225.

36. M. Kutter and F. A. P. Petitcolas, SPIE Security and Watermarking of Multimedia Contents, 1999, pp. 226-239.

37. Special Issue, Proc. IEEE 87(7) (1999).

38. W. Zhu, Z. Xiong, and Y. Q. Zhang, IEEE Trans. Circuits Syst. Video Technol. 9(4), 545-550 (1999).

39. Proc. Int. Workshop Inf. Hiding.

40. Special Issue, IEEE J. Selected Areas Commun. (May 1998).

41. M. D. Swanson, M. Kobayashi, and A. H. Tewfik, Proc. IEEE 86(6), 1,064-1,087 (1998).

42. G. C. Langelaar et al., IEEE Signal Process. Mag. 17(5), 20-46 (2000).

43. C. Podilchuk and E. Delp, IEEE Signal Process Mag. 18(4), 33-46 (2001)

\section{DISPLAY CHARACTERIZATION}

\author{
DAVID H. BRAINARD \\ University of Pennsylvania \\ Philadelphia, PA \\ Denis G. Pelli \\ New York University \\ New York, NY \\ Tom RoBson \\ Cambridge Research \\ Systems, Ltd. \\ Rochester, Kent, UK
}

\section{INTRODUCTION}

This article describes the characterization and use of computer-controlled displays. ${ }^{1}$ Most imaging devices are

\footnotetext{
1 The display literature often distinguishes between calibration and characterization (e.g. 1-3), calibration refers to the process of adjusting a device to a desired configuration, and characterization refers to modeling the device and measuring its properties to allow accurate rendering. We adopt this nomenclature here.
}

now computer controlled, and this makes it possible for the computer to take into account the properties of the imaging device to achieve the intended image. We emphasize CRT (cathode ray tube) monitors and begin with the standard model of CRT imaging. We then show how this model may be used to render the desired visual image accurately from its numerical representation. We discuss the domain of validity of the standard CRT model. The model makes several assumptions about monitor performance that are usually valid but can fail for certain images and CRTs. We explain how to detect such failures and how to cope with them.

Here we address primarily users who will be doing accurate imaging on a CRT. Inexpensive color CRT monitors can provide spatial and temporal resolutions of at least $1024 \times 768$ pixels and $85 \mathrm{~Hz}$, and the emitted intensity is almost perfectly independent of viewing angle. CRTs are very well suited for accurate rendering. Our treatment of LCDs (liquid crystal displays) is brief, in part because this technology is changing very rapidly and in part because the strong dependence of emitted light on viewing angle in current LCD displays is a great obstacle to accurate rendering. Plasma displays seem more promising in this regard.

We present all the steps of a basic characterization that will suffice for most readers and cite the literature for the fancier wrinkles that some readers may need, so that all readers may render their images accurately. The treatment emphasizes accuracy both in color and in space. Our standard of accuracy is visual equivalence: substituting the desired for the actual stimulus would not affect the observer. ${ }^{2}$ We review the display characteristics that need to be taken into account to present an arbitrary spatiotemporal image accurately, that is, luminance and chromaticity as a function of space and time. We also treat a number of topics of interest to the vision scientist who requires precise control of a displayed stimulus.

The International Color Consortium (ICC, http://www. color.org/) has published a standard file format (4) for storing "profile" information about any imaging device. ${ }^{3}$ It is becoming routine to use such profiles to achieve accurate imaging (e.g. by using the popular Photoshop ${ }^{\circledR}$ program). ${ }^{4}$ The widespread support for profiles allows most users to achieve characterization and correction without needing to understand the underlying characteristics of the imaging device. ICC monitor profiles use the standard CRT model presented in this article. For applications where the standard CRT model and instrumentation designed for the mass market are sufficiently accurate, users can simply buy a characterization package consisting of a program

\footnotetext{
${ }^{2}$ The International Color Consortium (4) calls this "absolute colorimetric" rendering intent, which they distinguish from their default "perceptual" rendering intent. Their "perceptual" intent specifies that "the full gamut of the image is compressed or expanded to fill the gamut of the destination device. Gray balance is preserved but colorimetric accuracy might not be preserved."

${ }^{3}$ On Apple computers, ICC profiles are called "ColorSync" profiles because the ICC standard was based on ColorSync. Free C source code is available to read and write ICC profiles $(5,6)$.

${ }^{4}$ Photoshop is a trademark of Adobe Systems Inc.
} 
and a simple colorimeter that automatically produces ICC profiles for their monitors. ${ }^{5}$ The ICC-required data in a monitor profile are just enough ${ }^{6}$ to specify the standard CRT model, as presented here. The ICC standard also allows extra data to be included in the profile, making it possible to specify extended versions of the standard CRT model and other relevant aspects of the monitor (e.g., modulation transfer function and geometry).

This article explains the standard CRT model (and necessary basic colorimetry) and describes simple visual tests (available online at http://psychtoolbox.org/tips /displaytest.html) that establish the model's validity for your monitor. Then, we show how to use the standard model to characterize your display for accurate rendering. Finally, the Discussion briefly presents several more advanced topics, including characterization of non-CRT displays. ${ }^{7}$

\section{CRT MONITOR BASICS}

We begin with a few basic ideas about the way CRT monitors produce light and computers control displays. The light emitted from each location on a monitor is produced when an electron beam excites a phosphor coating at the front of the monitor. The electron beam scans the monitor faceplate rapidly in a raster pattern (left to right, top to bottom), and the intensity of the beam is modulated during the scan so that the amount of light varies with the spatial position on the faceplate. It is helpful to think of the screen faceplate as being divided up into contiguous discrete pixels. The video voltage controlling the beam intensity is usually generated by a graphics card, which emits a new voltage on every tick of its pixel clock (e.g., $100 \mathrm{MHz}$ ). The duration of each voltage sample (e.g., $10 \mathrm{ns)} \mathrm{determines} \mathrm{the} \mathrm{pixel's} \mathrm{width}$ (e.g., $0.3 \mathrm{~mm}$ ). Each pixel is a small fraction of a raster line painted on the phosphor as the beam sweeps from left to right. The pixel height is the spacing of raster lines. Most monitors today are "multisync," allowing the parameters of the raster (pixels per line, lines per frame, and frames per second) to be determined by the graphics card. Color monitors contain three interleaved phosphor types (red, green, and blue) periodically arranged as dots or stripes across the face of the monitor. There are three electron beams and a shadow mask arranged so that each beam illuminates only one of the three phosphor types.

\footnotetext{
${ }^{5}$ Vendors of such packages include Monaco (http://www.monacosys.com/) and ColorBlind (http://www.color.com/).

${ }^{6}$ Actually, as noted by Gill (7), it is ... "a pity that the media black point is not a mandatory part of the profile in the same way [that] the media white point is, since the lack of the black point makes absolute colorimetric profile interpretation inaccurate". Thus, before purchasing monitor characterization software, one should consider whether the software will include the optional media black point tag in the monitor profiles it produces. We have not found a precise definition of the term "media black point" in the ICC documentation, but we infer that it refers to a specification of the ambient light $A(\lambda)$ defined in Eq. (1) below.

${ }^{7}$ More detailed treatments of CRTs (e.g., 2,8-11; see 12), colorimetry (e.g., 13-15), and use of ICC profiles (3) may be found elsewhere.
}

The phosphor interleaving is generally much finer than a pixel, so that the fine red-green-blue dots or stripes are not resolved by the observer at typical viewing distances. We will not discuss it here, but for some applications, for example, rendering text, it is useful to take into account the tiny displacements of the red, green, and blue subpixel components $(16,17)$.

The stimulus for color is light. The light from each pixel may be thought of as a mixture of the light emitted by the red, green, and blue phosphors. Denote the spectrum of the light emitted from a single monitor pixel by $C(\lambda)$. Then,

$$
C(\lambda)=r R(\lambda)+g G(\lambda)+b B(\lambda)+A(\lambda),
$$

where $\lambda$ represents wavelength, $R(\lambda), G(\lambda)$, and $B(\lambda)$ are the spectra of light emitted by each of the monitor's phosphors when they are maximally excited by the electron beam, $r, g$, and $b$ are real numbers in the range $[0,1]$, and $A(\lambda)$ is the ambient (or "flare") light emitted (or reflected) by the monitor when the video voltage input to the monitor is zero for each phosphor. We refer to the values $r, g$, and $b$ as the phosphor light intensities. ${ }^{8}$

Later, we discuss the kind of equipment one can use to measure light, but bear in mind that the characterization of a display should be based on the same light as the observer will see. Thus the light sensor should generally be in approximately the same position as the observer's eye. Happily, the luminance of CRTs is almost perfectly independent of viewing angle (i.e., it is nearly a lambertian light source), allowing characterization from one viewing point to apply to a wide range. (LCDs lack this desirable property. From a fixed head position, you can see variations in hue across a flat panel caused by the different viewing angles of the nearer and farther parts. This is a serious obstacle to characterization of LCDs for accurate rendering unless the viewpoint can be fixed. Plasma displays are much better in this regard.)

Note that Eq. (1) depends on the assumption of channel constancy: to a very good approximation, the relative spectrum of light emitted by each (R, G, or B) monitor channel is independent of the degree to which it is excited. ${ }^{9}$ To simplify the main development below, we assume that $A(\lambda)=0$. Correcting for nonzero ambient is

${ }^{8}$ Spectrally, Eq. (1) says that the light is a linear combination of contributions from the three phosphors. Similarly, spatially, the light measured at a particular pixel location is a linear combination of contributions from several pixels. The weighting of the contributions is the point-spread function. The point spread may be neglected while characterizing the display's color properties if you use a uniform test patch that is much bigger than the point spread. Thus, Eq. (1) does not take into account blur introduced by the optics of the electron beam and the finite size of the phosphor dots: the intensities $r, g$, and $b$ describe the total light intensity emitted at a pixel but not how this light is spread spatially. Treatment of the point-spread function is provided in the Discussion (see Modulation Transfer Function).

${ }^{9}$ Strictly speaking, Eq. (1) only requires phosphor constancy, the assumption that the relative spectrum of light emitted by each of the CRT's phosphors is invariant. It is possible for phosphor constancy to hold and channel constancy to be violated, for example, when the degree to which electrons intended for one 


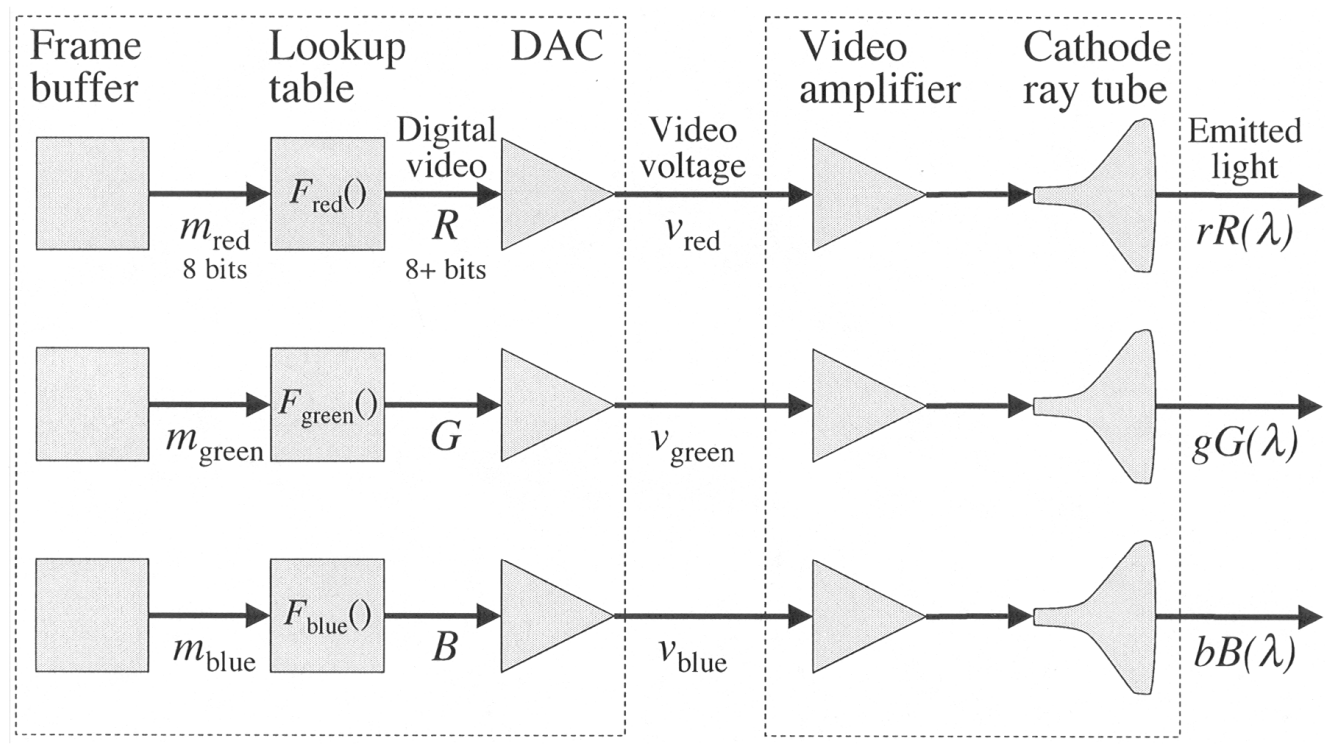

Figure 1. Schematic of graphics card and CRT monitor. The figure illustrates the video chain from digital pixel value to emitted light. As mentioned in the text and illustrated in Fig. 2, most graphics cards can run either in 24-bit mode, in which $m_{\text {red }}, m_{\text {green }}$, and $m_{\text {blue }}$ are independent, or in 8-bit mode, in which $m_{\text {red }}=m_{\text {green }}=m_{\text {blue }}$. The digital video output of each lookup table is 8 bits in most commercial graphics cards, but a few cards have more than 8 bits to achieve finer steps at the digital-to-analog converter (DAC) output. See color insert.

both straightforward and recommended, as explained in the Discussion.

Figure 1 shows how a pixel is processed. The graphics card generates the video voltages based on values stored in the onboard memory. Typically, user software can write digital values into two components of graphics card memory: a frame buffer and a color lookup table. The top panel of Fig. 2 illustrates the operation of the frame buffer and lookup table for what is referred to as "true color" or 24-bit ("millions of colors") mode. ${ }^{10}$ Three 8-bit bytes in the frame buffer are allocated for each monitor pixel. As shown in the figure, this memory can be thought of as three image planes, each arranged in a spatial grid corresponding to the layout of the monitor pixels. These are labeled Red, Green, and Blue in the figure. Each image plane controls the light emitted by one monitor phosphor. Consider a single pixel. The 8-bit (256 level) value $m_{\text {red }}$ in the Red image plane is used as an index to the red lookup table $F_{\text {red }}()$. This table has 256 entries, and each entry specifies the digital video value $R$ used to generate the video voltage $v_{\text {red }}$ that goes to the monitor to control the intensity of the electron beam as it excites the red phosphor at the pixel of interest. Similar indirection is used to obtain the digital video values $G$ and $B$ for the

phosphor dot excite another varies with digital video value (see, e.g., 1; also 18,19). For the presentation here, it is more convenient not to distinguish explicitly between phosphor and channel constancy.

10 This mode is also sometimes referred to as 32-bit mode: the 24 bits of data per pixel are usually stored in 32-bit words because this alignment provides faster memory access on most computers. green and blue phosphors:

$$
\begin{aligned}
& R=F_{\text {red }}\left(m_{\text {red }}\right), \\
& G=F_{\text {green }}\left(m_{\text {green }}\right), \\
& B=F_{\text {blue }}\left(m_{\text {blue }}\right) .
\end{aligned}
$$

In most graphics cards sold today, $R, G$, and $B$ are 8-bit numbers driving 8-bit digital-to-analog converters (DACs). This is very coarse compared to the 12- to 14-bit analog-todigital converters used in practically all image scanners sold today. It is odd that the digital imaging industry does not allow us to display with the same fidelity as we capture. However, some graphics cards for demanding applications such as radiology and vision science, do provide higher precision more-than-8-bit DACs, using the lookup table $F$ to transform the 8-bit values of $m_{\text {red }}, m_{\text {green }}$, and $m_{\text {blue }}$ into values of $R, G$, and $B$ selected from a palette of more finely quantized numbers.

The numbers $R, G$, and $B$ drive the graphic card's digital-to-analog converters, which produce video voltages proportional to the numbers, except for small errors in the digital-to-analog conversion:

$$
\begin{aligned}
v_{\text {red }} & =R+e_{\text {red }}\left(R, R^{\prime}\right), \\
v_{\text {green }} & =G+e_{\text {green }}\left(G, G^{\prime}\right), \\
v_{\text {blue }} & =B+e_{\text {blue }}\left(B, B^{\prime}\right),
\end{aligned}
$$

where $v$ represents voltage on a dimensionless scale proportional to the actual voltage, $e$ is the error, and $R^{\prime}, G^{\prime}$, and $B^{\prime}$ are the values of $R, G$, and $B$ for the immediately preceding pixel in the raster scan. The error $e$ has a static and a dynamic component. The static error depends 


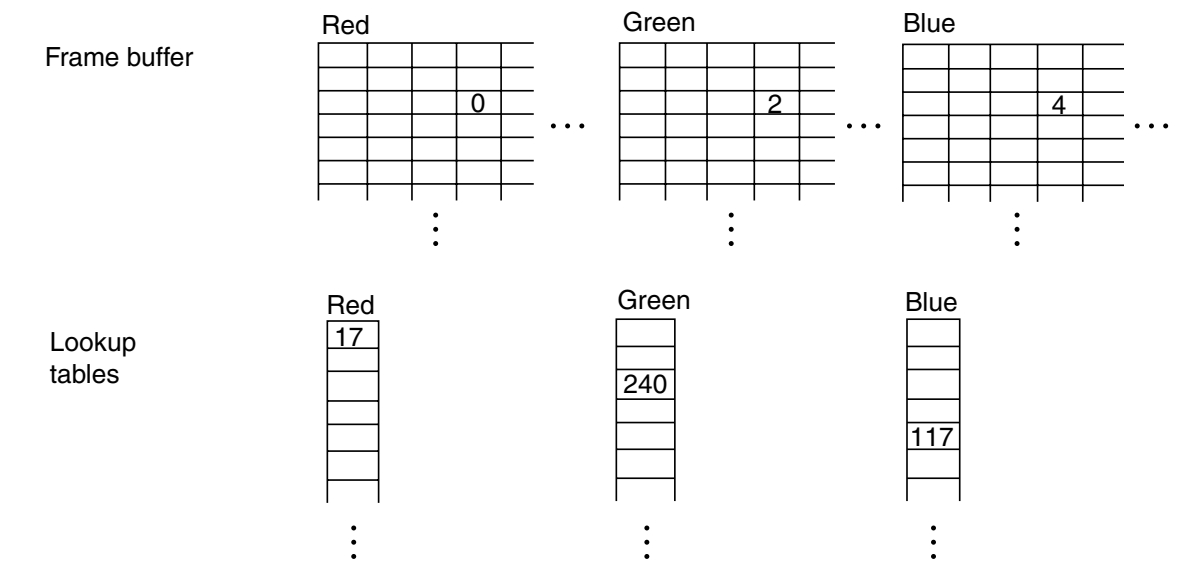

Frame buffer

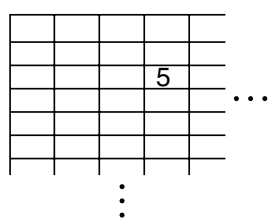

Color lookup

table

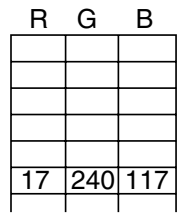

:

Figure 2. Graphics card operation. Top panel: 24-bit mode. The frame buffer may be thought of as three separate planes, one each for the red, green, and blue channels. Each plane allows specification of an 8-bit number (0-255) for each image location. In the figure, values for the pixel at $x=4, y=3$ are shown: these are 0 for the red plane, 2 for the green plane, and 4 for the blue plane. For each plane, the frame buffer value is used as an index to a lookup table. A frame buffer value of 0 indexes the first entry of the lookup table, and a frame buffer value of 255 indexes the last entry. Thus the configuration shown in the figure causes digital RGB values $(17,240,117)$ to be sent to the DACs for the pixel at $x=4, y=3$ Bottom panel: 8 -bit mode. The frame buffer is a single image plane, allowing specification of a single 8-bit number for each image location. This number is used to index to a color lookup table that provides the RGB values to be sent to the DACs. The 8-bit configuration shown in the bottom panel displays the same color for the pixel at $x=4, y=3$ as the 24-bit configuration shown in the top panel.

only on the current number and is sometimes specified by the manufacturer. It is generally less than one-half of the smallest step, that is, \pm 0.5 in the dimensionless scale we are using here (e.g., 0-255 for 8-bit DACs). The dynamic error is called a "glitch" and is a brief (few ns) error that depends on the relationship between the current value and that immediately preceding. The glitch is caused by the change of state of the switches (one per bit) inside the digital-to-analog converter. The glitch has a fixed duration, whereas the pixel duration is determined by the pixel clock rate, so the contribution of the glitch to the light intensity is proportionally reduced as the pixel duration is increased. The glitch is usually negligible.
The three video voltages produced by the graphics card, as described in Eq. (3), are transmitted by a cable to the CRT. Within the monitor, a video amplifier drives a cathode ray tube that emits light. The light emitted by the phosphors is proportional to the electron beam intensity, but that intensity is nonlinearly related to the video voltage, so the light intensity is a nonlinear function of video voltage:

$$
\begin{aligned}
& r=f_{\text {red }}\left(v_{\text {red }}\right), \\
& g=f_{\text {green }}\left(v_{\text {green }}\right), \\
& b=f_{\text {blue }}\left(v_{\text {blue }}\right),
\end{aligned}
$$


where $r, g$, and $b$ have the same meaning as in Eq. (1) and $f_{\text {red }}(), f_{\text {green }}()$, and $f_{\text {blue }}()$ are so-called gamma functions ${ }^{11}$ that characterize the input-output relationship for each monitor primary.

Note that the term "gamma function" is often used today to describe the single-pixel nonlinear transformation of many display devices, including printers. There are differences in both the form of the nonlinearities and the mechanisms that cause them. The gamma function of a CRT is caused by a space charge effect in the neck of the tube, whereas the gamma function of printers is usually caused by the spreading and overlapping of ink dots and the scatter of light within the paper. Different models are needed to account satisfactorily for the nonlinearities of different devices. Some analog-input LCD displays and projectors process the incoming signal electronically to provide a nonlinear response that approximates that of a traditional CRT, but they are being displaced by newer digital-input LCDs.

Equations (3) and (4) incorporate further assumptions about monitor performance, including assumptions of pixel independence and channel independence. These assumptions, particularly pixel independence, greatly simplify the CRT model and make it practical to invert it.

Pixel independence is the assumption that the phosphor light intensities $(r, g$, and $b)$ at each pixel depend solely on the digital video values for that pixel, independent of the digital video values for other pixels. Recall that, as a matter of convenience, Eq. (1) defines the phosphor intensities $r, g$, and $b$ before blur by the point-spread function of the CRT. The real blurry pixels overlap, but pixel independence guarantees that the contribution of each pixel to the final image may be calculated independently of the other pixels. The final image is the sum of all of the blurry pixels (21).

Channel independence is the assumption that the light intensity for one phosphor channel depends solely on the digital video value for that channel, independent of the digital video values for the other channels. The validity of these assumptions is discussed later.

There is a second common mode for graphics cards. This is typically referred to as "indexed color" or 8-bit ("256 colors") mode. It is illustrated at the bottom of Fig. 2. Here, there is only a single plane of 8-bit frame buffer values. These index to a single color lookup table to obtain $R$, $G$, and $B$ values; at each pixel, the same index value is used for all three channels so that $m_{\text {red }}=m_{\text {green }}=m_{\text {blue }}$. Thus, for 8-bit mode, only 256 colors (distinct $R, G, B$ combinations) are available to paint each frame. It is usually possible to load an entirely new lookup table for

11 The term gamma function is also used to describe the relationship between phosphor intensities (i.e., $r, g$, and $b$ ) and digital video values (i.e., $R, G$, and $B$ ) because this latter relationship is often the one measured. It is called a "gamma function" because the relationship has traditionally been described by power-law like functions where the symbol gamma denotes the exponent, for example, $r \propto\left[\left(R-R_{0}\right) /\left(255-R_{0}\right)\right]^{\gamma}$ for $R>R_{0}$ and $r=0$, otherwise [see Eq. (16) later]. Gamma is usually in the range 2 to 3 , with a value of 2.3 quoted as typical (11; see also 20). each frame. Most current graphic cards can be configured to operate in either 8- or 24-bit mode. The advantage of 8bit mode is that images can be moved into the frame buffer more rapidly, because less data must be transferred. The disadvantage is that only 256 colors are available for each frame. Both 8- and 24-bit modes are important and widely used in visual psychophysics (see 22).

Although it is useful to understand the difference between 8- and 24-bit graphics modes, the distinction is not crucial here. The digital part of the CRT video chain is simple and does not introduce distortions. As illustrated by Fig. 3, the key point is that, for each pixel, we must compute the appropriate $R, G$, and $B$ values to produce arbitrary desired phosphor intensities $r, g$, and $b$. This computation relies on measurements of the analog portion of the video chain and on the properties of the display. In particular, it is necessary to characterize the spectral properties of the light emitted by the monitor phosphors [Eq. (1)] and the gamma functions [Eqs. (3) and (4)].

\section{BASIC COLORIMETRY}

Equation (1) shows that monitors can produce only a very limited set of spectra $C(\lambda)$, those that consist of a weighted sum of the three fixed primaries. But that is enough because human color vision is mediated by three classes of light-sensitive cones, referred to as the $L, M$, and $S$ cones (most sensitive to long, middle, and short wavelengths, respectively). The response of each class of cones to a spectrum incident at the eye depends on the rate at which the cone pigment absorbs photons, and this absorption rate may be computed via the spectral sensitivity of that class of cones. Denote the spectral sensitivities of the $L$, $M$, and $S$ cones as $L(\lambda), M(\lambda)$, and $S(\lambda)$, respectively. Then the quantal absorption rates $l, m$, and $s$ of the $L, M$, and $S$ cones for a color stimulus whose spectrum is $C(\lambda)$ are given by the integrals

$$
\begin{gathered}
l=\int_{380 \mathrm{~nm}}^{780 \mathrm{~nm}} L(\lambda) C(\lambda) d \lambda, \\
m=\int_{380 \mathrm{~nm}}^{780 \mathrm{~nm}} M(\lambda) C(\lambda) d \lambda, \\
s=\int_{380 \mathrm{~nm}}^{780 \mathrm{~nm}} S(\lambda) C(\lambda) d \lambda,
\end{gathered}
$$

where each integral is taken across the visible spectrum, approximately $380 \mathrm{~nm}$ to $780 \mathrm{~nm}$. We refer to these quantal absorption rates as the cone coordinates of the spectrum $C(\lambda)$.

The integrals of Eq. (5) may be approximated by the sums

$$
\begin{gathered}
l=\sum_{i=1}^{n} L\left(\lambda_{i}\right) C\left(\lambda_{i}\right) \Delta \lambda, \\
m=\sum_{i=1}^{n} M\left(\lambda_{i}\right) C\left(\lambda_{i}\right) \Delta \lambda,
\end{gathered}
$$




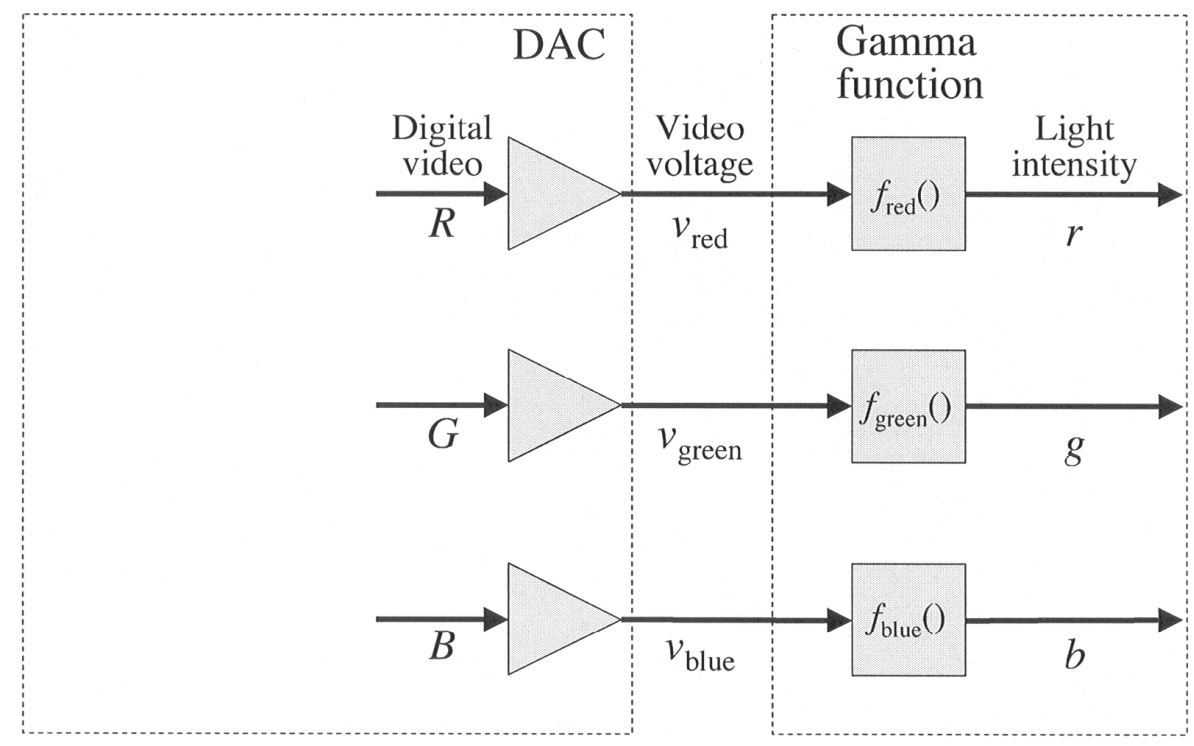

Figure 3. The standard CRT model. Based on Fig. 1, this reduced schematic shows the subsection of the video chain described by the standard CRT model. This subsection takes the digital video values $R, G$, and $B$ as input and produces intensities $r, g$, and $b$ as output. See color insert.

$$
s=\sum_{i=1}^{n} S\left(\lambda_{i}\right) C\left(\lambda_{i}\right) \Delta \lambda
$$

across wavelengths $\lambda_{i}$ evenly spaced across the visible spectrum, where $\Delta \lambda$ is the interval between wavelength samples. The CIE recommends sampling from $380 \mathrm{~nm}$ and $780 \mathrm{~nm}$ at $5 \mathrm{~nm}$ intervals, making $n=81$. Using matrix notation,

$$
\begin{aligned}
& \mathbf{s}=\left[\begin{array}{c}
l \\
m \\
s
\end{array}\right], \\
& \mathbf{S}=\left[\begin{array}{cc}
L\left(\lambda_{1}\right) & L\left(\lambda_{2}\right) \cdots \\
M\left(\lambda_{1}\right) & M\left(\lambda_{2}\right) \cdots \\
S\left(\lambda_{1}\right) & S\left(\lambda_{2}\right) \cdots
\end{array}\right] \Delta \lambda, \\
& \mathbf{c}=\left[\begin{array}{c}
C\left(\lambda_{1}\right) \\
C\left(\lambda_{2}\right) \\
\vdots
\end{array}\right],
\end{aligned}
$$

we can rewrite Eq. (6) as

$$
\mathbf{s}=\mathbf{S} \mathbf{c}
$$

Equation (8) computes cone coordinates $\mathbf{s}$ from the spectrum c of the color stimulus.

When two physically distinct lights that have the same spatial structure result in the same quantal absorption rates for the $L, M$, and $S$ cones, the lights are indistinguishable to cone-mediated vision. ${ }^{12}$ Thus, accurately rendering a desired image on a characterized monitor means choosing $R, G$, and $B$ values so that the spectrum c emitted by the monitor produces the same cone coordinates $\mathbf{s}$ as the desired image.

\footnotetext{
${ }^{12}$ We neglect for now a possible effect of rod signals that can occur at light levels typical of many color monitors. See Discussion: Use of Standard Colorimetric Data.
}

\section{CHARACTERIZATION USING THE STANDARD CRT MODEL}

The CRT model presented above is the standard CRT model used for color characterization (e.g., 1,2,23). ${ }^{13}$ Using the standard CRT model, we can find the digital video values $R, G$, and $B$ required to render a spectrum $C(\lambda)$ through the following computational steps:

1. Computing cone coordinates. Use Eq. (8) to find the $l, m$, and $s$ quantal absorption rates corresponding to $C(\lambda)$.

2. Computing phosphor light intensities. Find $r, g$, and $b$ such that the mixture expressed on the right side of Eq. (1) produces the same quantal absorption rates. This computation relies on measurements of the phosphor spectra but is independent of the gamma functions.

3. Gamma correction. Find DAC values $R, G$, and $B$ that will produce the desired phosphor intensities $r$, $g$, and $b$. This computation relies on measurements of the gamma functions but is independent of the phosphor spectra. Because each intensity $r, g$, and $b$ under the standard CRT model is a simple monotonic function of the corresponding digital video value $(R$, $G$, or $B$ ), it is straightforward to invert each gamma function to find the necessary digital video value to produce any desired output (assuming that the DAC error $e$ is negligible).

The measurements and computations required for each of these steps are discussed in more detail below. Note that before actually using the standard model, you will want to make sure it is valid for your monitor and stimuli.

13 Both Post (1) and Cowan (2) provide systematic developments of the standard model. Different authors have used different terms for the assumptions embodied by the standard model. Our nomenclature here is similar to Post's (1). 
Detecting failure of the standard CRT model and possible remedies are also discussed below.

Most CRT monitors have controls labeled "contrast" and "brightness" that affect the gamma function, the ambient light $A(\lambda)$, and the maximum luminance. These should be adjusted before beginning the characterization process. Write the date and "do not touch" next to the controls once they are adjusted satisfactorily.

\section{Computing Cone Coordinates}

Recall that, given the spectrum of a color stimulus c, Eq. (8) allows us to compute the cone coordinates s. Measuring spectra requires a spectroradiometer. But spectroradiometers are expensive, so it is more common to use a less expensive colorimeter to characterize the spectral properties of the desired light. A colorimeter measures the CIE XYZ tristimulus coordinates of light. $\mathrm{XYZ}$ tristimulus coordinates are closely related to cone coordinates. Indeed, the cone coordinates $\mathbf{s}$ of the light may be obtained, to a good approximation, by the linear transformation

$$
\mathbf{s}=\mathbf{T} \mathbf{x}
$$

where

$$
\mathbf{T}=\left[\begin{array}{ccc}
0.2420 & 0.8526 & -0.0445 \\
-0.3896 & 1.1601 & 0.0853 \\
0.0034 & -0.0018 & 0.5643
\end{array}\right]
$$

and

$$
\mathbf{x}=\left[\begin{array}{c}
X \\
Y \\
Z
\end{array}\right]
$$

The matrix $\mathbf{T}$ was calculated from the Smith-Pokorny estimates $(24,25)$ of cone spectral sensitivities, each cone sensitivity was normalized to a peak sensitivity of one, and the XYZ functions were as tabulated by the CIE $(26,27)$. The appropriate matrix may be easily computed for any set of cone spectral sensitivities (14). A detailed discussion of the relationship between cone coordinates and tristimulus coordinates is available elsewhere $(14,15)$.

\section{Computing Phosphor Intensities}

Adopting matrix notation for the phosphor spectra $\mathbf{P}$ and intensities $\mathbf{w}$,

$$
\begin{aligned}
& \mathbf{P}=\left[\begin{array}{ccc}
R\left(\lambda_{1}\right) & G\left(\lambda_{1}\right) & B\left(\lambda_{1}\right) \\
R\left(\lambda_{2}\right) & G\left(\lambda_{2}\right) & B\left(\lambda_{2}\right) \\
\vdots & \vdots & \vdots
\end{array}\right], \\
& \mathbf{w}=\left[\begin{array}{c}
r \\
g \\
b
\end{array}\right]
\end{aligned}
$$

allows us to rewrite Eq. (1) [neglecting $A(\lambda)$ ] as

$$
\mathbf{c}=\mathbf{P w}
$$

Using this to substitute for $\mathbf{c}$ in Eq. (8) yields

$$
\mathbf{s}=\mathbf{S P w}=\mathbf{M w},
$$

where the $3 \times 3$ matrix $\mathbf{M}$ equals $\mathbf{S} \mathbf{P}$. We can compute the matrix inverse of $\mathbf{M}$ and solve for $\mathbf{w}$,

$$
\mathbf{w}=\mathbf{M}^{-1} \mathbf{s}
$$

Equation (14) computes the phosphor intensities $\mathbf{w}(r, g$, and $b$ ) that will produce the desired cone coordinates $\mathbf{s}(l$, $m$, and $s$ ).

Calculation of Eq. (14) requires knowledge of the matrix $\mathbf{M}$,

$$
\begin{aligned}
\mathbf{M} & =\mathbf{S P} \\
& =\left[\begin{array}{lll}
\sum_{i=1}^{n} L\left(\lambda_{i}\right) R\left(\lambda_{i}\right) \Delta \lambda & \sum_{i=1}^{n} L\left(\lambda_{i}\right) G\left(\lambda_{i}\right) \Delta \lambda & \sum_{i=1}^{n} L\left(\lambda_{i}\right) B\left(\lambda_{i}\right) \Delta \lambda \\
\sum_{i=1}^{n} M\left(\lambda_{i}\right) R\left(\lambda_{i}\right) \Delta \lambda & \sum_{i=1}^{n} M\left(\lambda_{i}\right) G\left(\lambda_{i}\right) \Delta \lambda & \sum_{i=1}^{n} M\left(\lambda_{i}\right) B\left(\lambda_{i}\right) \Delta \lambda \\
\sum_{i=1}^{n} S\left(\lambda_{i}\right) R\left(\lambda_{i}\right) \Delta \lambda & \sum_{i=1}^{n} S\left(\lambda_{i}\right) G\left(\lambda_{i}\right) \Delta \lambda & \sum_{i=1}^{n} S\left(\lambda_{i}\right) B\left(\lambda_{i}\right) \Delta \lambda
\end{array}\right]
\end{aligned}
$$

Each element of $\mathbf{M}$ is the quantal absorption rate of one cone class for one phosphor spectrum at maximum excitation. Each column of $\mathbf{M}$ is the set of cone coordinates $\mathbf{s}$ of one phosphor spectrum [Eqs. 6 and 7]. Thus, to implement Eq. (14), it is necessary to know these cone coordinates for each phosphor. The best way to find them is to use a spectroradiometer to measure the phosphor emission spectra $R(\lambda), G(\lambda)$, and $B(\lambda)$. If such measurements are not feasible and only the XYZ tristimulus coordinates of each phosphor are available, then the matrix $\mathbf{M}$ may be computed using the relationship between the cone and tristimulus coordinates given in Eqs. (9) and (10).

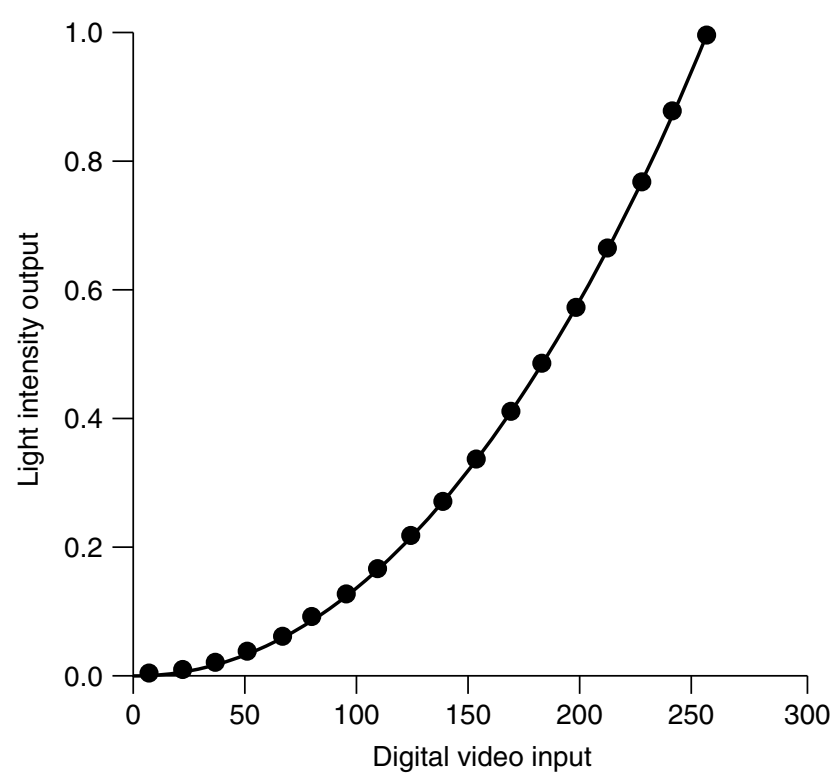

Figure 4. Typical gamma function. The plot shows the gamma function for a typical CRT monitor. The solid circles show measured data. The measurement procedure is described in the text. The intensity data were normalized to a maximum of one for a digital video value of 255 . The line shows the best fit obtained using Eq. (16), with parameter estimates $\gamma=2.11$ and $G_{0}=0$. 
Alternatively, if one prefers to specify color stimuli in terms of XYZ coordinates rather than cone coordinates, one may set $\mathbf{T}$ as the identity matrix and work directly in $\mathrm{XYZ}$ coordinates, starting with the colorimetric measurements and replacing the cone spectral sensitivities used to form the matrix $\mathbf{S}$ in the previous derivations by the CIE XYZ color matching functions.

\section{Gamma Correction}

Figure 4 shows a typical gamma function measurement. To obtain this curve, the spectrum of light emitted by the green phosphor was measured for 35 values of the $G$ digital video value where $R=B=0$. A measurement of the ambient light $(R=G=B=0)$ was subtracted from each individual measurement. Then, for each measured spectrum, a scalar value was found that expressed that spectrum as a fraction of the spectrum $G(\lambda)$ obtained from the maximum digital video value $(G=255)$. These scalars take on values between 0 and 1 and are the measured gamma function $f_{\text {green }}()$.

Given a desired value for the green-phosphor intensity $g$, gamma correction consists of using the measured gamma function to find the digital value $G$ that produces the best approximation to $g$. This is conceptually straightforward, but there are several steps.

The first step is to interpolate the measured values. Although exhaustive measurement is possible, fitting a parametric function to the measured values has the advantage of smoothing any error in the gamma function measurements. ${ }^{14}$ For most CRT monitors, measured gamma functions are well fit by the functional form (e.g., for the green phosphor)

$$
\begin{array}{ll}
g=f_{\text {green }}\left(G+e_{\text {green }}\right)=\left[\frac{\left(G-G_{0}\right)}{\left(255-G_{0}\right)}\right]^{\gamma}, & \text { for } G>G_{0} \\
g=f_{\text {green }}\left(G+e_{\text {green }}\right)=0, & \text { otherwise. }
\end{array}
$$

In Eq. (16), parameter $G_{0}$ represents a cutoff digital video value below which no incremental light is emitted, and parameter $\gamma$ describes the nonlinear form of the typical gamma function. The constant 255 in Eq. (16) normalizes the digital video value and is appropriate when $G$ is specified by 8-bit numbers. To be consistent with Eq. (4), we retain $e_{\text {green }}$, which is usually negligible. The curve through the data in Fig. 4 represents the best fit of Eq. (16) to the measured data. Note that Eq. (16) requires that phosphor intensity $g=0$ when digital video value $G=0$. This is appropriate because both our definition of phosphor intensities [Eq. (1)] and our measurement procedure (see above) account explicitly for the ambient light.

Equation (16) is easily inverted to determine, for example, $G$ from $g$ :

$$
G=\left(255-G_{0}\right) g^{1 / \gamma}+G_{0} .
$$

\footnotetext{
${ }^{14}$ In a demanding application using 8-bit DACs, it may be desirable to measure light intensity accurately for all 256 digital video values and to use the measured data directly instead of fitting a smooth function, so that the DAC's static error is taken into account in the gamma correction.
}

Because $G$ must be an integer and the value computed by Eq. (17) is real, the computed value of $G$ should be rounded up or down to minimize the error in $g$ predicted by Eq. (16).

\section{DETECTING FAILURES OF THE STANDARD CRT MODEL}

The standard CRT model is useful because it generally provides an acceptably accurate description of the performance of actual CRT monitors, because measurement of its parameters is straightforward, and because it is easily inverted. Use of the standard CRT model is implicit in the information stored in ICC monitor profiles: these profiles store information about the XYZ tristimulus coordinates and gamma function for each channel $(R, G$, and $B)$. For very accurate rendering, however, it is important to be aware of the assumptions that the model embodies and how these assumptions can fail. A number of authors provide detailed data on how far actual CRT monitors deviate from the assumptions of the standard CRT model $(1,2,18,19,21,28-31)$.

As noted above, the key assumption for the standard CRT model is pixel independence, expressed implicitly by Eqs. (3) and (4). Pixel independence is particularly important because, though it is easy to extend the standard CRT model to deal with dependent pixels, the extended model is hard to invert, making it impractical for fast image rendering. The next section (Extending the Standard CRT Model) briefly discusses other assumptions for the standard CRT model. It is generally possible to cope with the failures of these other assumptions because the standard CRT model can be extended to handle them in a fashion that still allows it to be inverted rapidly.

\section{Pixel Independence}

There are at least six causes for the failure of pixel independence (also see 21). Dependence on other pixels in the same frame (failures 1-4, below) has been called "spatial dependence", and dependence on pixels in preceding frames (failures 5 and 6 ) has been called "temporal dependence" (1). Failures 1 and 2 are short range, causing each pixel's color to depend somewhat on the preceding pixel in the raster scan. Failures 3-6 are long range in the current frame ( 3 and 4$)$ or in the preceding frames (5 and 6 ).

1. Glitch. As mentioned earlier, the glitch (the dynamic component of the DAC error) depends on the preceding as well as the current pixel. The DAC manufacturer generally specifies that the contribution of the glitch to the average voltage for the duration of a pixel at maximum clock rate is a fraction of a least significant step, so it is usually negligible.

2. Finite video bandwidth and slew rate. The whole video signal chain - including the DACs on the graphics card, the video cable, and the monitor's amplifiers - has a finite bandwidth so that it cannot instantly follow the desired voltage change from pixel to pixel, resulting in more gradual voltage 
transitions. Because this filtering effect precedes the nonlinear gamma function, it is not equivalent to simply blurring the final image horizontally along the raster lines. Smoothing or averaging before the nonlinear gamma function makes the final image dimmer. (Because the gamma function is accelerating, the average of two luminances produced by two voltages is greater than the luminance produced by the average voltage.) An excellent test for this effect is to compare the mean luminance of two fine gratings, one vertical and one horizontal $(21,29,31,32)$. This test is available on-line at http://psychtoolbox.org / tips / displaytest.html. Each grating consists of white and black one-pixel lines. Only the vertical grating is attenuated by the amplifier's bandwidth, so it is dimmer. Like the glitch, this effect can be reduced by slowing the pixel clock in the graphics card or, equivalently, by using two display pixels horizontally (and thus two clock cycles) to represent each sample in the desired image. Video bandwidth is normally specified by the manufacturer and should be considered when choosing a CRT monitor.

3. Poor high-voltage regulation. The electron beam current is accelerated by a high-voltage ( 15 to $50 \mathrm{kV})$ power supply, and on cheaper monitors, the voltage may slowly drop when the average beam current is high. This has the effect of making the intensity of each pixel dependent on the average intensity of all of the pixels that preceded it. (The highvoltage supply will generally recuperate between frames.) You can test for such long-distance effects by displaying a steady white bar in the center of your display surrounded by a uniform field of variable luminance. Changing the surround from white to black ideally would have no effect on the luminance of the bar. To try this informally without a photometer, create a cardboard shield with a hole smaller than the bar to occlude a flickering surround, and observe whether the bar is steady. This effect depends on position. The effect is negligible at the top of the screen and maximal at the bottom. A single high-voltage supply generally provides the current for all three channels $(R, G$, and $B)$, so that the effect on a particular test spot is independent of the channel used for background modulation. When the high voltage is very poorly regulated, the whole screen image expands as the image is made brighter, because as the increased current pulls the high voltage down, the electrons take longer to reach the screen and deflect more.

4. Incomplete DC restoration. Unfortunately, the video amplifier in most CRT monitors is not DC coupled (21). Instead it is AC coupled most of the time, and momentarily DC coupled to make zero volts produce black at the end of the vertical blanking interval. (DC, "direct current," refers to zero temporal frequency; AC, "alternating current," refers to all higher frequencies.) This is called "DC restoration," which is slightly cheaper to design and build than a fully DC coupled video circuit. If the AC time constant were much longer than a frame, the DC restoration would be equivalent to DC coupling, but, in practice, the AC time constant is typically short relative to the duration of a frame, so that the same video voltage will produce different screen luminances depending on the average voltage since the last blanking interval. As for failure 3, this effect is negligible at the top of the screen and maximal at the bottom. However, this effect can be distinguished from failure 3 by using silent substitution. To test, say, the green primary, use a green test spot, and switch the background (the rest of the screen) back and forth between green and blue. The green and blue backgrounds are indistinguishable to the high-voltage power supply (it serves all three guns) but are distinct to the video amplifiers (one per gun).

5. Temporal dependence. This is a special case of pixel dependence, where the pixel intensities depend on pixels in preceding frames. A typical example of this occurs when a full-field white screen is presented after a period of dark. Amongst other things, the large change in energy delivered to the screen results in distortions of the shadow mask and displayed luminances that are temporarily higher than desired. This effect may persist for several frames. It is normally difficult to characterize these phenomena precisely, especially in a general model, and the best line of defense is to avoid stimuli that stress the display. Measurement of temporal dependence requires an instrument that can measure accurately frame by frame.

6. Dynamic brightness stabilization. This is another cause of temporal dependence. Since the mid1990s, some CRT manufacturers (e.g., Eizo) have incorporated a new "feature" in their CRT monitors. The idea was to minimize objectionable flicker when switching between windows, by stabilizing the total brightness of the display. To this end, the monitor compensates for variations in the mean video voltage input to the display. This has the effect of making the intensity of each pixel dependent on the average voltage of all of the pixels in the preceding frame. This artifact, too, will be detected by the test described in 3 above. However, this effect is independent of spot position and thus can be distinguished from failures 3 and 4 , by testing at the top of the screen, where failures 3 and 4 are negligible.

Different monitors conform in different degrees to the standard CRT model. More expensive monitors tend to have higher video bandwidth and better regulation of the high-voltage power supply. If accuracy matters, it is worth performing tests like those described here to find a monitor whose performance is acceptable. A suite of test patterns for display in your web browser is available at http://psychtoolbox.org/tips / displaytest.html.

\section{Coping with Pixel Dependence}

The standard CRT model is easily extended to deal with dependent pixels, but the resulting model is hard to invert, 
making it impractical for many applications. However, an extended model may be useful in estimating the errors in your stimuli.

Once you understand the nature of pixel dependence, you may be able to minimize its effect by avoiding problematic stimuli. For example, the preceding pixel effects (failures 1 and 2 of pixel independence) can be reduced by reducing the rate of change of the video signal, either by spacing the changes out (e.g., double the viewing distance, and use a block of four pixels, $2 \times 2$, in place of each original pixel) or making a fine grating horizontal (parallel to the raster lines) instead of vertical. The spatialaverage effects (failures $3-6$ of pixel independence) can be reduced by maintaining a constant mean luminance during characterization and display. If the image to be displayed does not occupy the whole screen, then it is generally possible to mask a small section of the screen from view and to modulate the light emitted from this section to counterbalance modulations of the image that are visible. In this way, the total light output from the entire display may be held constant.

\section{EXTENDING THE STANDARD CRT MODEL}

\section{Channel Constancy}

As noted above, Eq. (1) embodies an assumption of channel constancy, that the relative spectrum emitted by an RGB monitor channel is independent of its level of excitation.

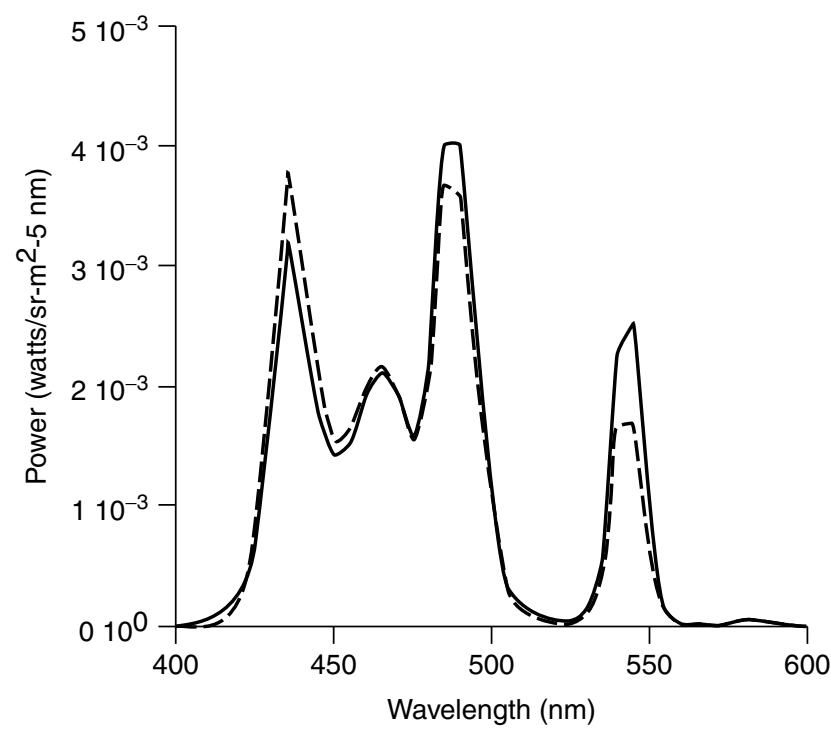

Figure 5. LCD primary spectra. A plot of the relative spectral power distribution of the blue primary of an LCD monitor (Marshall, V-LCD5V) in the spectral range 400-600 $\mathrm{nm}$ measured at two digital video values. Measurements were made in David Brainard's lab. The spectrum of the ambient light was subtracted from each measurement. The solid curve shows the spectrum for a digital video value of 255 . The dashed curve shows the spectrum for a digital video value of 28 , scaled to provide the best least-squares fit to the spectrum for a digital video value of 255 . The plot reveals a change in the relative spectrum of the emitted light as the digital video value changes from 28 to 255 . If there were no change, the two curves would coincide.
In our experience, channel constancy holds quite well for CRTs. However, for other display technologies, there can be significant violations of this assumption. Figure 5, for example, shows the relative spectrum of the green primary of an LCD monitor (see discussion of LCD displays later) at two digital video value levels, revealing a substantial change in the spectrum of this primary. It is possible to measure such changes systematically and correct for them. For example, Brainard et al. (33) describe a method based on using small-dimensional linear models to characterize spectral changes. This method was developed for handling spectral changes that occur when the voltage to filtered lamps is varied, but it also works well for LCD displays. Other authors have also proposed methods designed explicitly to deal with failures of channel constancy (28). Lookup table methods (see Channel Independence) may also be used to handle the effects of spectral changes.

\section{Channel Independence}

In Eq. (4), the form of the gamma function for the green phosphor is $g=f_{\text {green }}\left(v_{\text {green }}\right)$. A more general form for this function would be $g=f_{\text {green }}\left(v_{\text {red }}, v_{\text {green }}, v_{\text {blue }}\right)$, so that $g$ depends on its corresponding digital video value $G$ and also on $R$ and $B$. Failures of the simple form [Eq. (4)] are called failures of channel independence. Channel independence can fail because of inadequate power supply regulation (see item 3 under pixel independence above). Cowan and Rowell (18) and Brainard (19) describe procedures for testing for failures of channel independence.

Channel inconstancy and dependence go beyond the standard CRT color model but still obey pixel independence and are within the domain of a general color transformation model. Lookup tables may be used to characterize CRT monitors whose performance violates the assumptions of channel constancy and independence. To create a table, one measures the monitor output (e.g., $\mathrm{XYZ)} \mathrm{for} \mathrm{a} \mathrm{large} \mathrm{number} \mathrm{of} \mathrm{monitor} \mathrm{digital} \mathrm{video} \mathrm{value}$ triplets (i.e., RGB values). Then, interpolation is employed to invert the table and find the appropriate digital video value for any desired output. Cowan (2) provides an introduction to lookup table methods (see also 34,35). The ICC profile standard allows for storing and inverting a sampled representation of a general three-space to a three-space nonlinear transform.

\section{Spatial Homogeneity}

In general, all of the properties of a CRT may vary across the face of the CRT, and, for some applications, it may be necessary to measure this variation and correct for it. Luminance at the edge of a CRT is often $20 \%$ less than in the center (19). Failures of spatial homogeneity can be even larger for projectors. Brainard (19) found that the spatial inhomogeneity of a monitor could be characterized by a single light-attenuation factor at each location.

Spatial inhomogeneity does not violate pixel independence, because the light output at each pixel is still independent of the light output at other pixel locations. Spatial homogeneity may be handled by allowing the parameters of the standard model to depend on the pixel location. 


\section{Temporal Homogeneity}

There are two common causes of temporal variations in many types of display: those caused by warm-up and lifetime decay. CRTs and LCD panels (as a result of their backlight) take a significant time to warm up after being turned on. This can be long as 45 to 60 minutes during which time the luminance of a uniform patch will gradually increase by as much as $20 \%$. There may also be significant color changes during this period.

CRTs have finite lifetimes, partly because of browning of the phosphors by $\mathrm{X}$ rays. This causes the phosphors to absorb some light that would otherwise have been emitted. The same process affects the backlights used in LCD panels. The magnitude of this effect is proportional to the total light that has ever been emitted from the tube. Thus, presenting very bright stimuli for long periods will cause it to happen faster. Typical magnitudes of this phenomenon can be a $50 \%$ decrease in luminance across a few thousand hours.

In addition to an overall decrease in the amount of emitted light, repeated measurements of the gamma function of a 1989-vintage monochrome CRT monitor during its life revealed an increase in the cutoff parameter $G_{0}$ [Eq. (16)] by about six per month (on a scale of 0 to 255), when the monitor was left on continuously. We tentatively attribute this to loss of metal from the cathode, which may affect the MTF as well.

\section{DISCUSSION}

The preceding development has specified the steps of basic display characterization. Here we touch on several more advanced points.

\section{Further Characterization}

There are other imaging properties, beyond color transformation, that you may want to characterize.

Modulation Transfer Function (MTF). The optics of the electron beam and phosphor blur the displayed image somewhat. This is linear superposition and does not violate the pixel independence assumption. (The contribution of each blurry pixel may be calculated independently, and the final image is the sum of all of the blurry pixels.) Although it is easier to think of blur as convolution by a point spread, it is usually best to characterize it by measuring the Fourier analog of the point spread, the MTF. To do this, we recommend displaying drifting sinusoidal gratings and using a microphotometer to measure the light from a small slit (parallel to the gratings) on the display. (Your computer should read the photometer once per frame.) This should be done for a wide range of spatial frequencies. Adjust the drift rate to maintain a fixed temporal frequency, so that the results will not be affected by any temporal frequency dependence of the photometer. Results of such measurements are shown in Fig. 6. The monitor's MTF is given by the amplitude of the sinusoidal luminance signal as a function of spatial frequency. When the grating is horizontal (no modulation along individual raster lines), the blur is all optical and is well characterized as a linear system that has the specified MTF. When the grating is vertical, some of the "blur" is due to the finite video bandwidth (failure 2 of pixel independence, above), and because the effect is nonlinear, the MTF is only an approximate description.

Geometry. For some experiments, for example, judging symmetry, it may be important to produce images whose shape is accurately known. For this, you may wish to measure and correct for the geometric distortions across the face of your monitor (36). Some monitors allow adjusting of the geometry of the displayed image and such adjustment may be used to minimize spatial distortions.

\section{Ambient Light or "Flare"}

Correcting for the presence of ambient light [term $A(\lambda)$ in Eq. (1)] is easy. If we want to produce $C(\lambda)$, first we compute $C^{\prime}(\lambda)=C(\lambda)-A(\lambda)$ and simply proceed as described previously (see Characterizing with the Standard CRT Model), using $C^{\prime}(\lambda)$ in place of $C(\lambda)$. The same correction can also be applied if one starts with cone coordinates $\mathbf{s}$ or tristimulus coordinates $\mathbf{x}$. In these cases, one computes $\mathbf{s}^{\prime}$ or $\mathbf{x}^{\prime}$ by subtracting the coordinates of

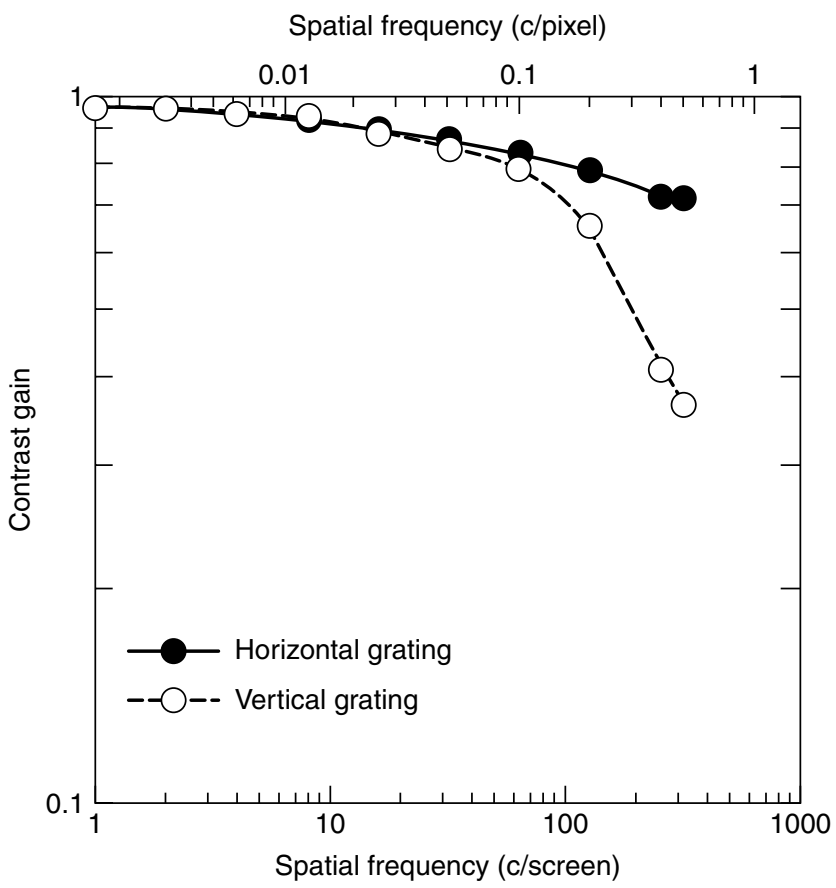

Figure 6. CRT monitor Modulation Transfer Function (MTF) The measured contrast of a drifting sinusoidal grating as a function of spatial frequency (normalized to 1 at zero frequency) for a CRT monitor, a 1989-vintage Apple High-Resolution Monochrome Monitor made by Sony. It has a fixed scan resolution of $640 \times 480$, a gamma of 2.28 , and a maximum luminance of $80 \mathrm{~cd} / \mathrm{m}^{2}$. As explained in the text, the contrast gain using horizontal gratings is independent of contrast, and thus is the MTF. The "contrast gain" using vertical gratings, normalized by the horizontal-grating result, characterizes the nonlinear effect of limited bandwidth of the video amplifier, which will depend on contrast. It is best to avoid presenting stimuli at frequencies at which the video amplifier limits performance, that is, where the dashed curve falls below the solid curve. 
the ambient light from the desired coordinates and then proceeds as before.

When a monitor viewed in an otherwise dark room has been adjusted to minimize emitted light when $R=G=$ $B=0$, and only a negligible fraction of its emission is reflected back to it from surfaces in the room, correcting for the ambient light is probably not necessary. Under other conditions, the correction can be quite important.

\section{What If I Care Only About Luminance?}

For many experiments it is desirable to use just one primary and set the others to zero. Or, because observers typically prefer white to tinted displays, you may wish to set $R=G=B$ and treat the display as monochrome, using a one-channel version of the standard CRT model.

\section{Fine Control of Contrast}

As noted above, both 8-bit and 24-bit modes on graphics cards usually use 8-bit DACs to produce the video voltages that drive the CRT. The 256-level quantization of the 8-bit DACs is too coarse to measure threshold on a uniform background. A few companies sell video cards that have more-than-8-bit DACs. ${ }^{15}$

There are several ways to obtain finer contrast steps using 8-bit DACs, but none is trivial to implement:

1. Use a half-silvered mirror to mix two monitors optically (37).

2. Optically mix a background light with the monitor image, for example, by using a slide projector to illuminate the face of the CRT (38).

3. For gray-scale patterns, add together the three video voltages produced by a graphics card to produce a single-channel signal that has finer steps (see 39). For color patterns, add together the corresponding $v_{\text {red }}, v_{\text {green }}$, and $v_{\text {blue }}$ voltages from two synchronized graphics cards. (Synchronizing monitors can be technically difficult, however.)

4. Move the observer far enough away so that individual pixels cannot be resolved and use dithering or error diffusion $(40,41)$.

5. Use a high frame rate, and dither over time.

\section{Use of Standard Colorimetric Data}

Standard estimates of color matching functions represent population averages for color normal observers and are typically provided for foveal viewing at high light levels. These estimates may not be appropriate for individual observers or particular viewing conditions. First, there is variation among observers in which lights match, even among color normal observers $(42,43$; see also 25,27,44-50). Second, the light levels produced by typical CRTs may not completely saturate the rods. If rods participate significantly in the visual processing of the stimulus, then color matches computed on the

\footnotetext{
${ }^{15}$ A list of such video cards may be found at http://psychtoolbox. org/tips/videocards.html.
}

basis of standard colorimetry will be imperfect for lights viewed outside the fovea. Some guidance is available for determining whether rods are likely to have an effect at typical CRT levels (see 51; also 13).

Unfortunately, correcting for individual variation is not trivial, because determining the appropriate color matching functions for a given individual and viewing conditions requires substantial measurement. If individual cone sensitivities or color matching functions are known, then it is straightforward to employ them by substituting them for the standard functions in the matrix $\mathbf{S}$ of Eq. (7). In some experiments, stimuli are presented on monitors to nonhuman observers. Here it is crucial to customize the rendering calculations for the spectral sensitivities appropriate for the species of interest (see 52).

For psychophysical experiments where taking the role of rods into account is critical, one can consider constructing custom four-primary displays, restricting stimuli to the rod-free fovea or employing an auxiliary bleaching or adapting light to minimize rod activity.

For experiments where silencing luminance is of primary interest, individual variation can be taken into account by using a psychophysical technique such as flicker photometry to equate the luminance of stimuli that diller in chromaticity (15).

\section{Other Display Devices}

Although CRT monitors remain the dominant technology for soft copy display, they are by no means the only display technology. Liquid crystal display (LCD) devices are becoming increasingly popular and in fact are the dominant display technology used in laptop computers and data projection systems. Other emerging technologies include the digital light processor (DLP; 53) and plasma displays. Printers, obviously, are also important.

Characterization of other display devices requires applying the same visual criterion of accuracy. For each type of display device, a model of its performance must be developed and evaluated. The standard CRT model is a reasonable point of departure for LCD, DLP, and plasma displays. We discuss these in more detail below.

Printers are more difficult to characterize. The standard CRT model does not provide an acceptable description of printers. First, printers employ subtractive (adding ink causes more light to be absorbed) rather than additive color mixture, so that Eq. (1) does not hold. Second, the absorption spectrum of a mixture of inks is not easy to predict from the absorption spectra of individual inks. Third, the spectrum of reflected light depends on the inks laid down by the printer and also on the spectrum of the ambient illumination. The illumination under which the printed paper will be viewed is often not under the control of the person who makes the print. Lookup tables (see above) are generally employed to characterize the relationship between digital values input to the printer and the reflected light, given a particular reference illuminant. Accordingly, ICC printer profiles provide for specification of tabular data to characterize printer performance. A detailed treatment of printer characterization is beyond the scope of this article (see 2,3,35,54,55). 
LCD Panels. LCDs appear commonly in two manifestations: as flat panel equivalents of the CRT for desktop and laptop computers and as the light control element in projection displays. When designed to replace monitors, some manufacturers have tried to imbue them with some of the characteristics of CRT displays, so LCDs have typically accepted the same analog video signal from the computer that is used to drive a CRT. However, since the 1999 publication of the Digital Visual

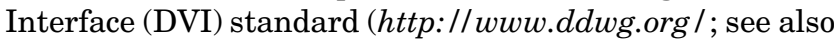
http://www.dell.com/us / en / arm / topics / vectors_2000dvi.htm), a rapidly increasing proportion of LCD displays accept digital input. At least in principle, digital-input LCDs can provide independent digital control of each pixel and promise excellent pixel independence. LCDs can be characterized similarly to CRT displays, but bear in mind the following points:

1. Angular dependence. The optical filtering properties of LCD panels can have a strong angular dependence, so it is important to consider the observer's viewing position when characterizing LCD displays. This is especially important if the observer will be off-axis or there will be multiple observers. Angular dependence may be the greatest obstacle to the use of LCDs for accurate rendering.

2. Temporal dependencies. The temporal response of LCD panels can be sluggish, resulting in more severe violations of the assumption of temporal independence than typically observed in CRT displays. For example, when measuring the gamma function, a different result will be obtained if one measures the output for each digital video value after a reasonable settling time than if one sandwiches one frame at the test level between two frames of full output. It is important to try to match the timing of the characterization procedure to the timing of the target display configuration.

3. Warm-up. The LCD panel achieves color by filtering a backlight normally provided by a cold-cathode fluorescent display. These behave similarly to CRTs when warming up, so be prepared to wait for 45 minutes after turning one on before expecting consistent characterization.

4. Channel constancy. As illustrated by Fig. 5, some LCD displays do not exhibit channel constancy. This does not appear to be inherent in LCD technology, however. Measurements of other LCD panels $(56,57)$ indicate reasonable channel constancy, at least as assessed by examining variation in the relative values of $\mathrm{XYZ}$ tristimulus coordinates.

5. Output timing. It can be important to know when the light is emitted from the display with respect to generating video signals. In a CRT-based display, the video signal modulates the electron beam directly, so it is easy to establish that the light from one frame is emitted in a $10 \mathrm{~ms}$ burst (assuming a $100 \mathrm{~Hz}$ frame rate) starting somewhere near the time of the frame synchronization pulse and the top of the screen, but this need not be so in an LCD system. To evaluate the output timing, arrange to display a single light frame followed by about ten dark frames. Connect one of the video signals (say the Green) to one channel of an oscilloscope and connect the other channel to a photodiode placed near the top of the screen. (Note that no electronics are needed to use the photodiode but the signal may be inverted and the voltage produced is logarithmically, rather than linearly, related to the incident light.) When observing a CRT, it will be possible to identify a short pulse of light about $1 \mathrm{~ms}$ or so wide located somewhere near the beginning of the video stream. If the detector is moved down the screen, the pulse of light will move correspondingly toward the end of the video frame. When observing an LCD panel, the signals look completely different. The light pulse is no longer a pulse but a frame-length block, and there may be a significant delay between the video stream and the arrival of the light. In fact, in some displays, the two may have no fixed relationship at all (see Fig. 7).

6. Resolution. Analog-input LCD panels (and projectors) contain interlace electronics that automatically resample the video signal and interpret it in a manner suitable for their own internal resolution and refresh rate. This is desirable for easy interface to different computers, but the resampling can introduce both spatial and temporal dependencies that make accurate imaging more difficult. If possible, LCD displays should be run at their native spatial and temporal resolution. Even then, it is not guaranteed that the electronics pass the video signal unaltered, and one should be alert for spatial and temporal dependencies. This consideration also applies to DLP displays.

7. Internal quantization. Analog-input LCDs may actually digitize the incoming video voltages with a resolution of only 6 or 8 bits before displaying it, so be prepared to observe quantization beyond that created by the graphics card.

8. Gamma. There is no inherent mechanism in an LCD panel to create the power-law nonlinearity of a CRT; therefore a power-type function [e.g., Eq. (16)] does not work very well to describe the function relating digital video value to phosphor light intensity. One way to deal with this is to make measurements of the light out for every possible digital video valve in and invert the function numerically.

LCD Projectors. Analog-input LCD displays currently offer little advantage in terms of image quality over a CRT display, but the unique problems posed by some environments such as the intense magnetic fields inside a MRI scanner mandate the use of a projector. Apart from CRT-based projectors, there are two types of projection displays commonly available: those based on LCDs, and those based on digital light processors (DLPs).

LCD projectors have properties very similar to LCD panels except that the light source is often an arc lamp rather than a cold-cathode fluorescent device, and the pixel processing is more elaborate. Therefore, the same 


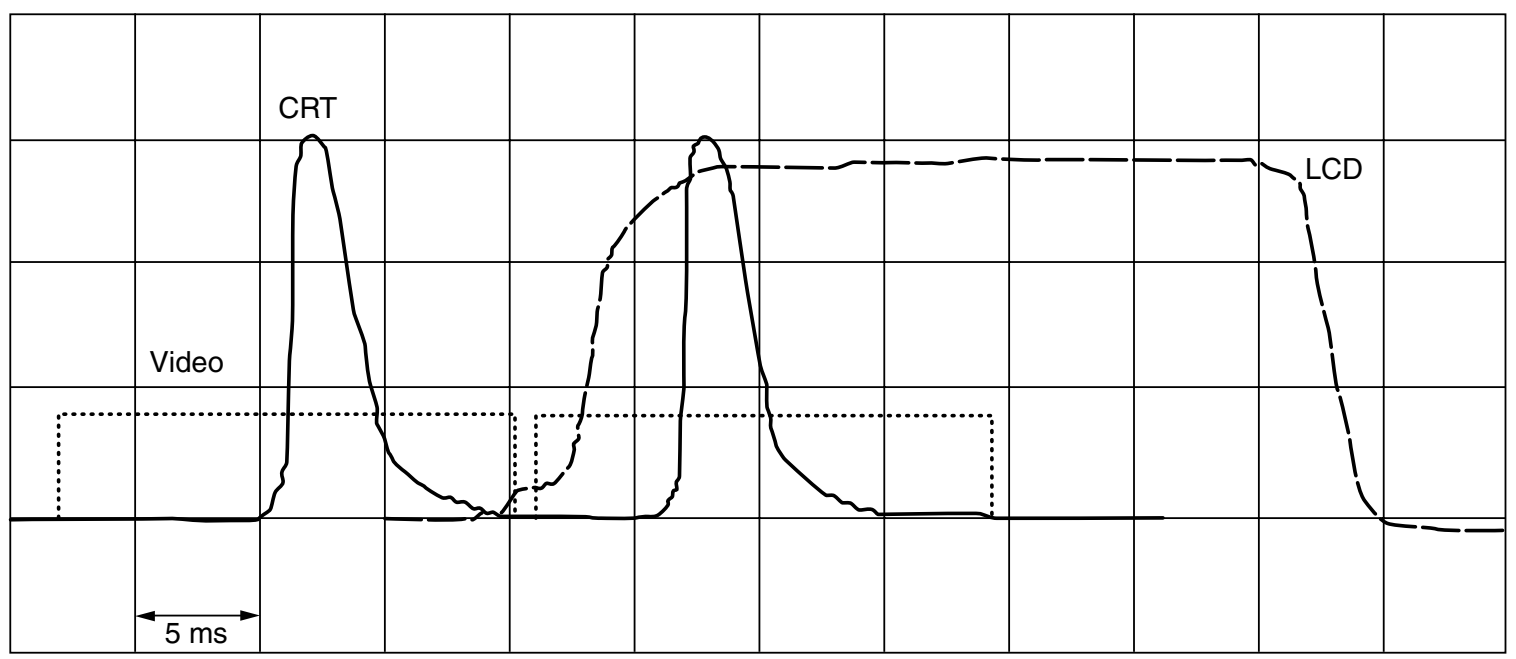

Figure 7. Delay in LCD projection. Diagram showing how the light output from the center of a projection screen, illuminated by an analog-input LCD projector, varies with time when the patch is nominally illuminated for two frames before being turned off. This is compared to the light from a CRT driven by the same video signal. Note the extra 12-ms delay imposed by the LCD projector's electronics.

considerations that apply to LCD panels generally apply to LCD projectors as well. Projectors are often used in conjunction with "gain" screens, which have nonlambertian viewing characteristics, and as for LCD panels, the light that reaches the observer varies with viewing position.

DLP Projectors. Digital light processing (DLP) projectors work by using a two-dimensional array of tiny mirrors (digital micromirrors) that can be deflected by about $20^{\circ}$ at high speed; $15 \mu$ s switching times are usual. These are used as light valves to direct the light from a high-intensity source either through the projection lens or off to the side. A color display is generated either by using three of these devices to deflect colored light filtered from the main source (as in modern LCD projectors) or by using one array operating at three times the net frame rate to deflect colored light provided by a rotating filter wheel. Typically, this rotates at about $150 \mathrm{~Hz}$ to give a $50 \mathrm{~Hz}$ display. The picture breakup that occurs when the eye makes saccadic movements makes projectors based on the filter wheel design difficult to use.

Currently three-mirror DLP projectors are quite expensive in comparison to LCD projectors, and many are designed for large-scale projection (e.g., digital cinema). Packer et al. (58) evaluate how well a three-mirror DLP device conforms to a number of the assumptions of the standard CRT model.

Plasma. Plasma displays are much more expensive than CRTs and LCDs but are currently favored by museums (e.g., the Museum of Modern Art and the Metropolitan Museum of Art in New York City) because the plasma display is both flat like an LCD and nearly lambertian (i.e., emitted light is independent of viewing angle) like a CRT.

\section{Instrumental Considerations}

Three types of instrument are typically used to characterize display devices: spectroradiometers, colorimeters, and photometers. Spectroradiometers measure the full spectral power distribution of light. Colorimeters typically allow measuring of CIE XYZ tristimulus coordinates. Photometers measure only luminance (proportional to CIE Y). Zalewski (59) provides a detailed treatment of light measurement. Several sources $(1,11,60,61$; http://psychtoolbox.org/tips/lightmeasure.html) discuss instrumentation for display characterization.

A spectroradiometer provides the most general characterization of the spectral properties of a display. One may compute cone coordinates or XYZ tristimulus coordinates from the spectral power distributions measured by spectroradiometers. If one wishes to use a description of color vision that differs from that specified by the CIE system (e.g., 24,25,27,42,62,63), then it is necessary to characterize the display by using a spectroradiometer. However, spectroradiometers are expensive (thousands of dollars), whereas colorimeters often cost less than a thousand dollars and the XYZ measurements they provide are sufficient for many applications. Photometers measure only luminance and thus are suitable only for applications where the color of the stimuli need not be characterized.

How much instrumental precision is required? The answer, of course, depends on the application. A simple calculation can often be used to convert the effect of instrumental error into a form more directly relevant to a given application. As an example, suppose that we wish to modulate a uniform field so that only one of the three classes of cones is stimulated, and the modulation is invisible to the other two. Such "silent substitution" techniques are commonly used in visual psychophysics to allow isolation of individual mechanisms (e.g., individual cone types; see 64,65). Figure 8 shows an 


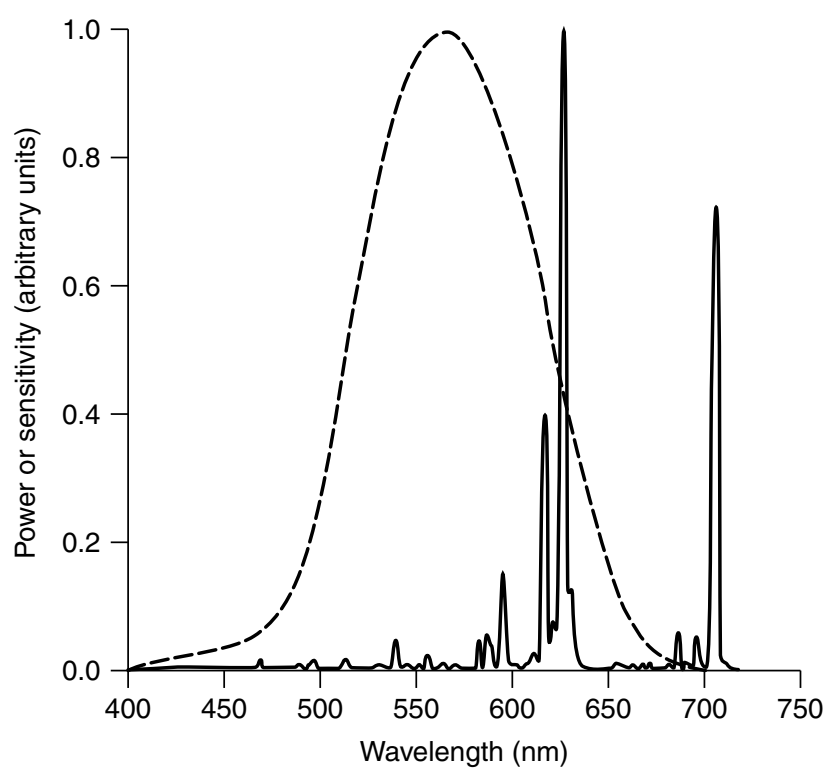

Figure 8. CRT red primary emission compared with L cone sensitivity. The solid line is a plot of the spectral power distribution of a typical CRT red primary. The measurements were made in David Brainard's lab and have approximately 1-nm resolution. The dashed line is a plot of an estimate (24) of the human L cone spectral sensitivity. Both curves have been normalized to a maximum of 1 . Note that considerable light power is concentrated in a region where the slope of the $\mathrm{L}$ cone sensitivity is steep.

estimate of the spectral sensitivity of the human L cone along with the spectral power distribution of a typical CRT red phosphor emission. Considerable phosphor power is concentrated in the spectral interval where the slope of the L cone sensitivity function is steep. One might imagine that calculations of the $\mathrm{L}$ cone response to light from this phosphor are quite sensitive to imprecisions in the phosphor measurement. To investigate, we proceeded as follows. We started with measurements of a CRT's phosphor emissions that had roughly a 1-nm resolution. Then, we simulated two types of imprecision. To simulate a loss of spectral resolution, we convolved the initial spectra using a Gaussian kernel $5 \mathrm{~nm}$ wide (standard deviation). To simulate an error in spectral calibration, we shifted the spectra $2 \mathrm{~nm}$ toward the longer wavelengths. We computed three stimulus modulations for each set of simulated measurements. Each modulation was designed to generate $20 \%$ contrast for one cone type and silence the other two. Then, we used the original spectral measurements to compute the actual effect of the simulated modulations. The effect is very small for the reduced resolution case. The maximum magnitude of modulation in cone classes that should be silenced is $0.25 \%$. However, the 2-nm wavelength shift had a larger effect. Here, a nominally silenced cone class can see almost $2 \%$ contrast. For this application, spectral calibration is more critical than spectral resolution.

One point that is often overlooked when considering the accuracy of colorimeters and photometers is how well the instrument's spectral sensitivities match those of their target functions: $X, Y$, and $Z$ color matching functions for colorimeters or luminance sensitivity $(Y)$ for photometers. To characterize the accuracy of a photometer, for example, it is typical to weight differences between instrumental and luminance spectral sensitivity at each wavelength in proportion to luminance sensitivity at that wavelength. This means that the specified accuracy is predominantly affected by discrepancies between instrumental and luminance sensitivity in the middle of the visible spectrum, where luminance sensitivity is high. The specified accuracy is not very sensitive to discrepancies in the short- or long-wavelength regions of the spectrum, where luminance sensitivity is low. A photometer that is specified to have good agreement with the luminance sensitivity function will accurately measure the luminance of a broadband source, but it may perform very poorly when measurements are made of spectrally narrower light, such as that emitted by CRT phosphors. The indicated luminance in such a situation can be wrong by $50 \%$ or more.

A final question that arises when using any type of light measuring instrument for a CRT is whether the instrument was designed to measure pulsatile sources. The very short bursts of light emitted as the raster scans the faceplate of the CRT have peak intensities of 10 to 100 times the average intensity. This can distort the result obtained by using electronics not designed for this possibility.

\section{Recommended Software}

ICC. For many purposes, sufficiently accurate imaging may be obtained simply by using any available commercial package to characterize the CRT and create an ICC profile and then using an ICC-compatible image display program.

Vision Research. After using a wide variety of software packages (e.g., see directories 66,67) for running vision experiments, we have come to the conclusion that it is very desirable that the top level, in which the experimenter designs new experiments, be a full-fledged language, preferably interactive, like BASIC or MATLAB. Writing software from scratch is hard. Some packages overcome this limitation by allowing users to design experiments by just filling out a form rather than actually programming. However, this limits the experiment to what the author of the form had in mind and makes it impossible to do a really new experiment. The best approach seems to be to program in a general purpose language and write as little new code as possible for each new experiment by modifying the most similar existing program. In that spirit, the free Psychophysics Toolbox (http://psychtoolbox.org/) provides a rich set of extensions to MATLAB to allow precise control and synthesis of visual stimuli within a full-featured interactive programming environment, along with a suite of demo programs $(68,69)$. Psychophysica (http://vision.arc.nasa.gov/mathematica/psychophysica/), also free, provides similar extensions for Mathematica (70).

Rush - "Hogging" the Machine. Today's popular computers interrupt the user's program frequently to grant 
time to other processes. This is how the computer creates the illusion of doing everything at once. The difficulty with this is that the interruptions can cause unwanted pauses in the temporal presentation of a stimulus sequence. Although it is difficult to shut down the interrupting processes completely, we have found it both possible and desirable to suppress interrupts, hogging the machine, for the few seconds it takes to present a visual stimulus. In the Psychophysics Toolbox (see above), this facility is called Rush and allows a bit of MATLAB code to run at high priority without interruption.

\section{SUMMARY}

Today's graphics cards and CRT monitors offer a cheap, stable, and well-understood technology suited to accurate rendering after characterization in terms of a simple standard model. This article describes the standard model and how to use it to characterize CRT displays. Certain stimuli will strain the assumption of pixel independence, but it is easy to test for such failures and often possible to avoid them. New technologies, particularly LCD, DLP, and plasma displays, are emerging as alternatives to CRT displays. Because these technologies are less mature, there are not yet standard models available for characterizing them. Development of such models can employ the same rendering intent and color vision theory introduced here, but the specifics of the appropriate device model are likely to differ. Digital-input LCD displays promise excellent pixel independence but may be difficult to use for accurate rendering because of the high dependence of the emitted light on the viewing angle.

\section{Acknowledgments}

We thank J. M. Foley, G. Horwitz, A. W. Ingling, T. Newman, and two anonymous reviewers for helpful comments on the manuscript. More generally, we learned much about monitors and their characterization from W. Cowan, J. G. Robson, and B. A. Wandell. This work was supported by grant EY10016 to David Brainard and EY04432 to Denis Pelli.

\section{BIBLIOGRAPHY}

1. D. L. Post, in H. Widdel and D. L. Post, eds., Color in Electronic Displays, Plenum, NY, 1992, pp. 299-312.

2. W. B. Cowan, in M. Bass, ed., Handbook of Optics, vol. 1, Fundamentals, Techniques, and Design, McGraw-Hill, NY, 1995, pp. 27.1-27.44.

3. R. Adams and J. Weisberg, GATF Practical Guide to Color Management, Graphic Arts Technical Foundation, Sewickley, PA 1998.

4. ICC, Specification ICC.1:1998-09 file format for color profiles, 1998, http://www.color.org / profiles.html.

5. G. Gill, ICC File I/O README, http://web.access.net.au/ argyll/color.html.

6. D. Wallner, Building ICC Profiles-The Mechanics and Engineering, 2000, http://www.color.org / iccprofiles.html.

7. G. Gill, What's wrong with the ICC profile format anyway? 1999, http://web.access.net.au/argyll/icc_problems.html.

8. D. E. Pearson, Transmission and Display of Pictorial Information, Pentech Press, Wiley, New York, 1975.
9. P. A. Keller, The Cathode-Ray Tube: Technology, History, and Applications, Palisades Press, NY, 1991.

10. T. R. H. Wheeler and M. G. Clark, in H. Widdel and D. L. Post, eds., Color in Electronic Displays, Plenum Press, NY, 1992, pp. 221-256.

11. P. A. Keller, Electronic Display Measurement-Concepts, Techniques and Instrumentation, J Wiley, NY, 1997.

12. D. H. Brainard and D. G. Pelli, Raster graphics psychophysics bibliography, http://psychtoolbox.org/tips/rasterbib.html.

13. G. Wyszecki and W. S. Stiles, Color Science-Concepts and Methods, Quantitative Data and Formulae, 2nd ed., J Wiley, NY, 1982.

14. D. H. Brainard, in M. Bass, ed., Handbook of Optics, vol. 1, Fundamentals, Techniques, and Design, McGraw-Hill, NY, 1995, pp. 26.1-26.54.

15. P. K. Kaiser and R. M. Boynton, Human Color Vision, 2nd ed., Optical Society of America, Washington, DC, 1996.

16. S. Daly, Soc. Inf. Display 2001 Dig. XXXII, 1,200-1,203 (2001)

17. R. A. Tyrell, T. B. Pasquale, T. Aten, and E. L. Francis, Soc. Inf. Display 2001 Dig. XXXII, 1,205-1,207 (2001).

18. W. B. Cowan and N. Rowell, Color Res. Appl. Suppl. 11, S33-S38 (1986)

19. D. H. Brainard, Color Res. Appl. 14, 23-34 (1989).

20. C. Poynton, Frequently-asked questions about gamma, http://www.inforamp.net/ poynton / GammaFAQ.html.

21. D. G. Pelli, Spatial Vision 10, 443-446 (1997); http://vision. nyu.edu/VideoToolbox/PixelIndependence.html.

22. A. B. Watson et al., Behav. Res. Methods Instrum. Comp. 18 587-594 (1986).

23. B. A. Wandell, Foundations of Vision, Sinauer, Sunderland, MA, 1995.

24. V. Smith and J. Pokorny, Vision Res. 15, 161-171 (1975).

25. P. DeMarco, J. Pokorny, and V. C. Smith, J. Opt. Soc. A 9, 1,465-1,476 (1992).

26. CIE, Colorimetry, 2nd ed., Bureau Central de la CIE, VIENNA, 1986, publication 15.2.

27. A. Stockman and L. T. Sharpe, Color and vision database, http://cvision.ucsd.edu/index.htm.

28. D. L. Post and C. S. Calhoun, Color Res. Appl. 14, 172-186 (1989).

29. N. P. Lyons and J. E. Farrell, Soc. Inf. Display Int. Sym. Tech. Dig. 20, 220-223 (1989).

30. R. S. Berns, R. J. Motta, and M. E. Gorzynski, Color Res. Appl. 18, 299-314 (1993).

31. A. C. Naiman and W. Makous, SPIE Conf. Human Vision Visual Process. Digital Display, III, 1666, 1992, 41-56.

32. Q. J. Hu and S. A. Klein, Soc. Inf. Display 94 Dig. 25, 19-22 (1994).

33. D. H. Brainard, W. A. Brunt, and J. M. Speigle, J. Opt. Soc. Am. A 14, 2,091-2,110 (1997).

34. E. S. Olds, W. B. Cowan, and P. Jolicoeur, J. Opt. Soc. A 16, $1,501-1,505$ (1999).

35. P. C. Hung, J. Electron. Imaging 2, 53-61 (1993).

36. L. T. Maloney and K. Koh, Behav. Res. Methods Instrum. Comput. 20, 372-389 (1988).

37. C. C. Chen, J. M. Foley, and D. H. Brainard, Vision Res. 40, $773-788(2000)$

38. A. B. Poirson and B. A. Wandell, Vision Res. 36, 515-526 (1996).

39. D. G. Pelli and L. Zhang, Vision Res. 31, 1,337-1,350 (1991). 
40. C. W. Tyler, Spatial Vision 10, 369-77 (1997).

41. J. B. Mulligan and L. S. Stone, J. Opt. Soc. Am. A 6, 1,217-1,227 (1989)

42. W. S. Stiles and J. M. Burch, Optica Acta 6, 1-26 (1958).

43. M. A. Webster and D. I. A. MacLeod, J. Opt. Soc. Am. A 5, 1,722-1,735 (1988).

44. J. Pokorny, V. C. Smith, G. Verriest, and A. J. L. G. Pinckers, Congenital and Acquired Color Vision Defects, Grune and Stratton, NY, 1979.

45. J. Nathans et al., Science 232, 203-210 (1986).

46. J. Neitz and G. H. Jacobs, Vision Res. 30, 621-636 (1990).

47. J. Winderickx et al., Nature 356, 431-433 (1992).

48. J. Neitz, M. Neitz, and G. H. Jacobs, Vision Res. 33, 117-122 (1993).

49. M. Neitz and J. Neitz, Science 267, 1,013-1,016 (1995).

50. J. Carroll, C. McMahon, M. Neitz, and J. Neitz, J. Opt. Soc. A 17, 499-509 (2000).

51. A. G. Shapiro, J. Pokorny, and V. C. Smith, J. Opt. Soc. Am. A 13, 2,319-2,328 (1996).

52. L. J. Fleishman et al., Anim. Behav. 56, 1,035-1,040 (1998).

53. L. J. Hornbeck, Digital light processing for high-brightness high-resolution applications. Texas Instruments Report, 1997.

54. R. W. G. Hunt, The Reproduction of Colour, 4th ed., Fountain Press, Tolworth, England, 1987.

55. B. A. Wandell and L. D. Silverstein, in S. K. Shevell, ed. The Science of Color, 2nd ed., Optical Society of America, Washington, DC, 2001.

56. M. D. Fairchild and D. R. Wyble, Colorimetric characterization of the Apple Studio Display (Flat Panel LCD). Munsell Color Science Laboratory Report, Rochester Institute of Technology, Rochester, NY, 1998; http: //www.cis.rit.edu/mcsl/research/reports.shtml.

57. J. E. Gibson and M. D. Fairchild, Colorimetric characterization of three computer displays (LCD and CRT). Munsell Color Science Laboratory Report, Rochester Institute of Technology, Rochester, NY, 2001; http://www.cis.rit.edu/ mcsl/research/reports.shtml.

58. O. Packer et al., Vision Res. 41, 427-439 (2001).

59. E. F. Zalewski, in M. Bass, ed., Handbook of Optics, vol. 2, McGraw-Hill, NY, 1995, pp. 24.3-24.51.

60. R. S. Berns, M. E. Gorzynski, and R. J. Motta, Color Res. Appl. 18, 315-325 (1993).

61. D. H. Brainard and D. G. Pelli, Light measurement instrumentation, http://psychtoolbox.org / tips /lightmeasure.html.

62. J. J. Vos, Color Res. Appl. 3, 125-128 (1978).

63. A. Stockman, D. I. A. MacLeod, and N. E. Johnson, J. Opt. Soc. Am. A 10(12), 2,491-2,521 (1993).

64. O. Estévez and H. Spekreijse, Vision Res. 22, 681-691 (1982).

65. D. H. Brainard, in P. K. Kaiser and R. M. Boynton, eds., Human Color Vision, Optical Society of America, Washington, DC, 1996, pp. 563-579.

66. LTSN Psychology, http://www.psychology.ltsn.ac.uk/search_resources.html.

67. F. Florer, Powermac software for experimental psychology, http: // vision.nyu.edu / Tips / FaithsSoftwareReview.html.

68. D. H. Brainard, Spatial Vision 10, 433-436 (1997).

69. D. G. Pelli, Spatial Vision 10, 437-442 (1997).

70. A. B. Watson and J. A. Solomon, Spatial Vision 10, 447-466 (1997).

\section{DYE TRANSFER PRINTING TECHNOLOGY}

\author{
Nobuhito Matsushiro \\ Okidata Corporation \\ Gunma, Japan
}

\section{INTRODUCTION}

This article describes the structure and operation of dye-sublimation thermal-transfer printing technology. In addition to the structure and operation, the capabilities and limitations of the technology such as resolution, speed, intensity levels, color accuracy, and size are described. Though the subject of this chapter is the dye-sublimation printer, the details of other thermal transfer printers are included to assist in understanding and comparing the capabilities and limitations of the dye-sublimation printer.

\section{HISTORY OF PRINTERS USING DYE TRANSFER}

Dye-transfer printers form images on sheets using different types of energy such as mechanical pressure, heat, and light. Impact printers use mechanical pressure whereas nonimpact printers use heat and light. In this article, the histories of both impact and nonimpact printers are overviewed. Which is important to understand the position of the dye-sublimation printer in the history of dye-transfer printers. The history following includes both impact printers and nonimpact printers which include the dye-sublimation printer.

\section{Impact Printers Using Dye Transfer}

1960 Development of the horizontal chain line printer, the original line printer.

1961 The golf ball printer established the position of the serial impact printer.

1970 Development of the daisy wheel printer, a typeface-font serial impact printer, the successor to the golf ball printer.

1970 The dot matrix printer is the mainstay of current serial impact printers.

\section{Nonimpact Printers Using Dye Transfer}

1930s Development of wax-type thermal-transfer recording paper.

Development of the early sublimation dye transfer method.

1940s Development of heat-sensitive paper using almost colorless metallic salts.

1950s Commercialization of colorant melting by exposure to infrared radiation.

Disclosure of the patent for the thermal printer. Development of a prototype electrosensitive transfer printer.

1960s Development of the leuco-dye-based heatsensitive recording sheet, a current mainstay in the market.

1969 Development of the heat-mode laser-based transfer printer. 